\title{
Encounter based Routing in Opportunistic Networks
}

\author{
Nidhi $^{1}$, R. K. Chauhan ${ }^{2}$ \\ ${ }^{I}$ (P.G. Student, DCSA, Kurukshetra University Kurukshetra, INDIA) \\ ${ }^{2}$ (Professor, DCSA, Kurukshetra University Kurukshetra, INDIA)
}

\begin{abstract}
Opportunistic networks are wireless networks which may be used in areas where large delays are presented between data transmission also long distances may be handled by nodes. In these networks nodes may communicate with others through intermediate nodes. Data transmission takes place via various routing protocols. An opportunistic network provides standard routing protocols that are epidemic routing, prophet routing, spray and wait routing etc. In this paper these protocols are discussed with their own pros and cons. After that a comparative analysis between them is also presented.
\end{abstract}

Keywords: Opportunistic networks, epidemic, prophet, spray and wait, store-carry-forward and movement models etc.

\section{INTRODUCTION}

TCP/IP neglects to work appropriately or may even quit working totally when there is no path between source and destination. Due to such circumstances, a more up to date system has developed which worked in the environment in which no end to end path is present between nodes. This system is called as opportunistic Network (OPPNET). OPPNET is a discontinuously associated Network where the end-to-end ways may not exist and correspondence routes might just be accessible through time and versatility. Because of absence of reliable availability, OPPNET uses store carry and forward mechanism i.e., in the wake of accepting a few bundles, a node carried them until it contacts another node and afterward advances the messages. Since OPPNET routing depends on portable nodes to forward bundles for one another, the directing execution (e.g., the quantity of messages conveyed to their destinations) relies on upon whether the nodes interact with one another or not [1].

1.1 Characteristics in Opportunistic networks: There are various characteristics of OPPNET. Some of them are as follows:

\begin{tabular}{|c|c|}
\hline Intermittent connectivity & - No end to end path \\
\hline High Latency & $\begin{array}{l}\text { - Any two nodes may never meet each } \\
\text { other. }\end{array}$ \\
\hline Low Data Rate & - Due to the long latency of data delivery. \\
\hline Disconnection & - It is hard to find an end-to-end path. \\
\hline Long Queuing Delay & - Because of the disconnection. \\
\hline $\begin{array}{c}\text { Dynamic Network } \\
\text { Topology }\end{array}$ & $\begin{array}{l}\text { - Different types of user behavior will } \\
\text { result in dramatically different network } \\
\text { conditions. }\end{array}$ \\
\hline
\end{tabular}

Fig. 1: Characteristics of OPPNET 
1.2 Routing protocols in Opportunistic networks: OPPNET provides following routing protocols that uses store carry forward mechanism to forward the packets these are:

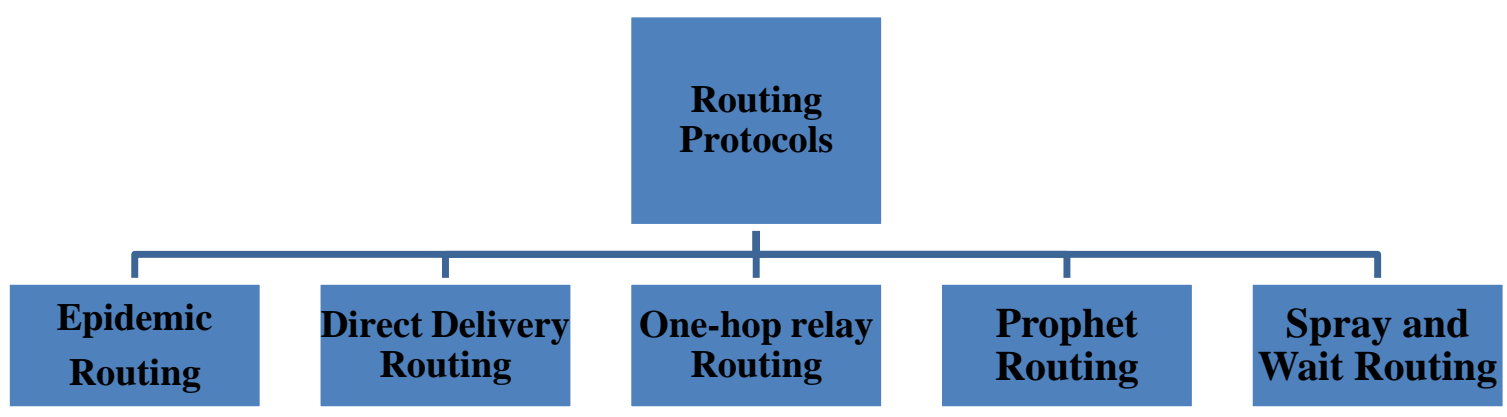

Fig. 2: Classifications of Various Routing Protocols in OPPNET

i. Epidemic routing: In epidemic routing whenever two nodes come across with one another then they replace all the messages currently they carried. When contact duration is over they hold the same set of messages. When this process repeated multiple times then packets are flooded in network so drawback of this scheme is that flooding creates overhead during transmission [2].

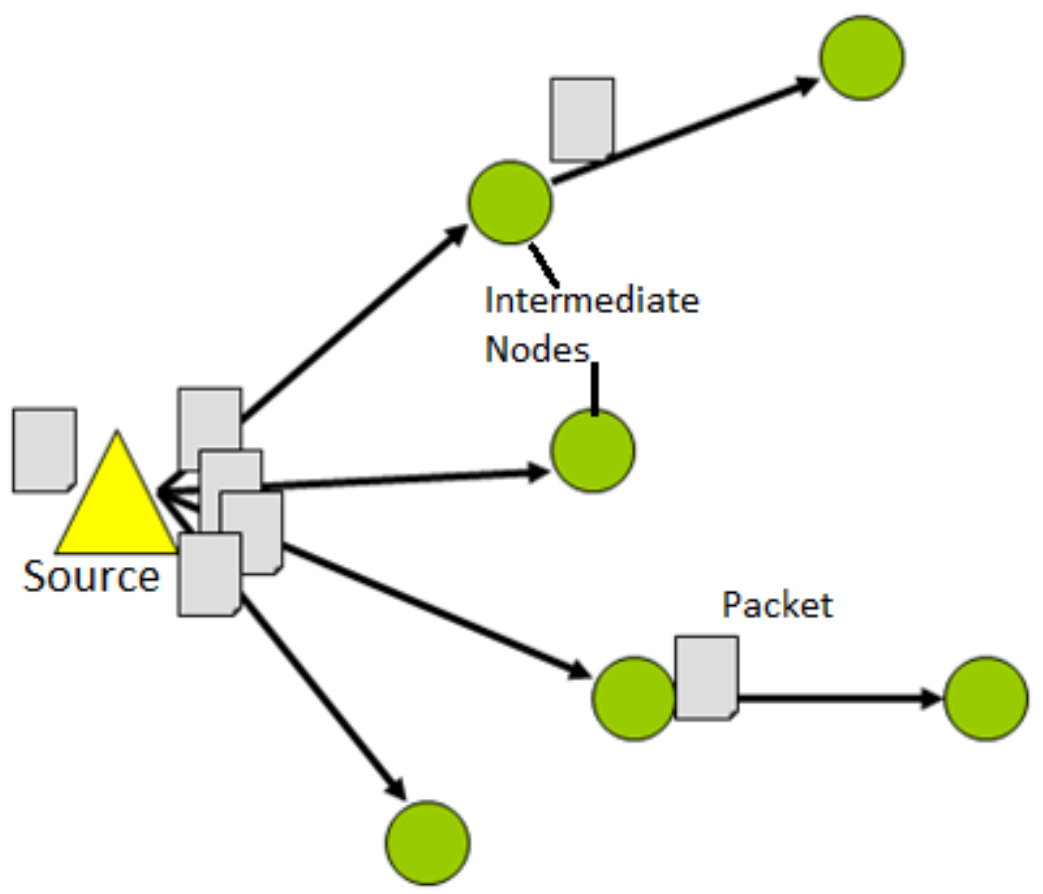

Fig. 3: Epidemic routing

ii. Direct delivery routing: This data delivery scheme is one of the simplest possible where a source delivers a packet to a destination when it comes in direct-contact [3].

iii. One-hop relay scheme: In this scheme, the source delivers a packet to an intermediate node, which in turn delivers the same to the destination. In one hop relay scheme one extra copy of messages are transmitted in network as compare to direct delivery routing [3].

iv. Prophet routing: Past contacts and Transitivity are used in prophet routing protocols to find the next forwarding node [3].

v. $\quad$ Spray and wait scheme: In this routing there are two phases:

Spray phase (only once): L message duplicates are at first spread to L unique nodes.

Wait phase: If the destination is not come to in the spray stage, the $\mathrm{L}$ nodes conveying a message duplicate perform direct transmission [6]. 


\section{RELATED WORK}

In [8] concentrated on the basic weakness of social-aware directing and sending plans in OPPNET. So as to survey framework delicacy from group structure perspective, we have proposed the CVA issue, broke down the minimization of NMI measure also, gave key bits of knowledge into the choice of hubs that are significant to the group structure.

In [9] studied opportunistic network architecture and investigated the social measurements from encounter, social elements and social properties, individually. They demonstrated that experience data is essential embodiment of social measurements in opportunistic networks. Social measurements, for example, social components and social properties, including social diagram properties what's more, group structure. We then expand the directing techniques from alternate points of view in like manner: experience based steering procedures, directing plans as per social includes and steering techniques in light of social properties.

In [10] proposed an routing mechanism where the transmission heading and the quantity of the duplicates are progressively controlled by data of the entire circulation rate of the nodes.

In [11] proposed content based message forwarding routing that uses predicates for nodes to promote their interests. Predicate parameter was used to indicate the delivery rate of messages. When nodes receive predicate parameter then they updates their predicate value after successful.

In [12] presented location based routing that abuses the normality implanted in human moving example. As human developments regularly display a high level of redundancy including consistent visits to specific spots furthermore, normal contacts amid day by day exercises, they predict a mobility of nodes for future by tracing movement of nodes.

In [13] authors performed comparative analysis on classical routing protocols of OPPNET. To analyze routing protocols authors uses performance measure parameters like delivery ratio, average number of hop count and overhead ratio to find out energy efficient routing protocol.

In [21] authors proposed SONR routing based on social aware routing that uses markov chain model to calculate delivery probability of each node and find optimal node with maximum delivery probability. Proposed protocols were compared by epidemic routing and spray and wait routing.

In [14] proposed routing protocols that uses the concept of cooperation called GAR. GAR incorporates a helpful message exchange plan and a cushion administration methodology. In the helpful message exchange plot, the constrained transmission capacity is considered and the message exchange needs are intended to boost the enhanced conveyance likelihood. In the cradle administration technique, by considering the imperative of support space, they proposed an agreeable message reserving plan and the dropping request of the messages is intended to minimize the diminished conveyance likelihood.

In [15] proposed a routing principle that uses mathematical model to find out optimal routing. Proposed scheme was based on social grouping in which data transmission take place when social interaction between nodes are high to forward the packets. Proposed scheme was energy efficient with low overhead ratio.

\section{PROBLEM FORMULATION}

The present research work focus on an algorithm to measure contacts between nodes and measure frequent nodes. EBR is the routing in which encounters between nodes are measured with the help of calculating contact between nodes occurs when two node come in range of each other and perform message transmission, calculating most frequent nodes, which are actively participating in message transmission process. Most frequent nodes have highest message transmission rate. By discovering contacts and frequent nodes we can easily improve delivery ratio and reduce replication of packets. 


\section{EVALUATION OF SOCIAL AWARE ROUTING PROTOCOLS}

In this section evaluation of existing routing strategies are presented with different parameters and their features based on social environment.

TABLE I ROUTING PROTOCOLS EVALUATION WITH NETWORK PERFORMANCE PARAMETERS

\begin{tabular}{|c|l|l|l|l|}
\hline Sr. No. & Routing protocols & Centrality & Similarity & Energy \\
\hline 1 & CAR & Present & Absent & Efficient \\
\hline 2 & BUBBLE & Present & Absent & Not efficient \\
\hline 3 & GAR & Present & Absent & Efficient \\
\hline 4 & SONR & Present & Absent & Efficient \\
\hline 5 & Prohet & Absent & Present & Not efficient \\
\hline 6 & MaxProphet & Absent & Present & Not efficient \\
\hline 7 & Simbet & Present & Present & Not efficient \\
\hline
\end{tabular}

In Table I evaluation of various existing routing protocols are presented. This evaluation was done using performance parameters like centrality, similarity and energy efficiency. Routing schemes such as CAR, SONR and GAR was energy efficient routing protocols.

In Table II routing protocols are classified based on their social features.

TABLE II ROUTING PROTOCOLS BASED ON SOCIAL FEATURES

\begin{tabular}{|c|l|l|l|}
\hline Sr. No. & \multicolumn{1}{|c|}{ Routing protocols } & Metric & \multicolumn{1}{|c|}{ Features } \\
\hline 1 & Social-aware routing & Awareness likeness & $\begin{array}{l}\text { Uses user enthusiasm to improve the } \\
\text { utilization of substance replication. }\end{array}$ \\
\hline 2 & Social greedy & community space & $\begin{array}{l}\text { Makes the information comparing so as to } \\
\text { send choices the social separation, which is } \\
\text { figured by the comparability of qualities. }\end{array}$ \\
\hline 3 & Homophily-based routing & Homophily & $\begin{array}{l}\text { Spreads most comparable information things } \\
\text { among companions and most distinctive } \\
\text { information things to outsiders. }\end{array}$ \\
\hline 4 & Social feature-based routing & community attributes & $\begin{array}{l}\text { Conducts hypercube and ascertain highlight } \\
\text { separation to quantify the closeness as } \\
\text { steering utility. }\end{array}$ \\
\hline
\end{tabular}




\section{CONCLUSION}

OPPNET provides various routing protocols like epidemic, prophet, spray and wait, direct delivery and first contact to forward data through intermediate nodes. In this paper these protocols are discussed. After that literature review of encounter based routing has been presented. In EBR various encounters occurred during data transmission are measure. On the basis of these encoders next forwarding nodes are selected. EBR improves delivery ratio while message delay increases because of measurement of encounters.

\section{REFERENCES}

[1] PeiyanYuan, LilinFan , PingLiu and ShaojieTang, Recent Progress In Routing Protocols of Mobile Opportunistic Networks: A Clear Taxonomy, Analysis and Evaluation, Journal of Network and Computer Applications Published by Elsevier Ltd , 2016, pp:1-8.

[2] Konglin Zhua, Wenzhong L, Xiaoming Fuc and Lin Zhang, Data Routing Strategies in Opportunistic Mobile Social Networks:Taxonomy and Open Challenges”, Computer Networks Published by Elsevier Ltd, 2015, pp. 1-15.

[3] J. Wu, Y. Wang, Opportunistic Mobile Social Networks, CRC Press, 2014.

[4] K. Zhu, W. Li and X. Fu, Rethinking Routing Information in Mobile Social Networks: Location-Based or SocialBased? Comput. Commun. 42, 2014, pp. 24-37.

[5] K. Zhu, W. Li and X. Fu, Smart: A Social and Mobile Aware Routing Strategy for Disruption Tolerant Networks, in Proceedings of IEEE Transactions on Vehicular Technology, 2014.

[6] Anju Rani, Atul Sharma, Dr. Dinesh Singh and Sanjeev Indora, Movement Models based Performance Comparison of Routing Protocols in DTN, in Proceedings of Second International Conference on Emerging Research in Computing, Information, Communication and Applications(ERCICA-14), 2014.

[7] Y. Wang and J. Wu, Social-tie-based Information Dissemination in Mobile Opportunistic Social Networks, in Proceedings of IEEE 14th International Symposium andWorkshops on aWorld ofWireless, Mobile and Multimedia Networks (WoWMoM), 2013, pp. 1-6.

[8] Y. Wang, W.S. Yang and J. Wu, Analysis of a Hypercube-Based Social Feature Multipath Routing in Delay Tolerant Networks, IEEE Trans. Parallel Distributed System, vol. 24 (9), 2013, pp. 1706-1716.

[9] W. Chang, J. Wu, C.C. Tan and F. Li, Sybil Defenses in Mobile Social Networks, in Proceedings of Global Telecommunications, GLOBECOM, 2013, pp. 641-646.

[10] R. Ciobanu, C. Dobre and V. Cristea, Sprint: Social Prediction-Based Opportunistic Routing, in Proceedings of 14th IEEE International Symposium andWorkshops on a World of Wireless, Mobile and Multimedia Networks, WoWMoM, 2013, pp. $1-7$.

[11] T. Abdelkader, K. Naik, A. Nayak, N. Goel and V. Srivastava, SGBR: a Routing Protocol For Delay Tolerant Networks Using Social Grouping, IEEE Trans.on Parallel Distrib. Syst. 24 (12), 2013, pp. 2472-2481.

[12] B. Ahlgren, C. Dannewitz, C. Imbrenda, D. Kutscher and B. Ohlman, A Survey of Information-Centric Networking, IEEE Commun. Mag. 50 (7), 2012, pp. 26-36.

[13] Cheng Gang1, SONG Mei, ZHANG Yong, XING Yi-Hai and BAO Xu-Yan, Routing Protocol Based on Social Characteristics Foropportunistic Networks, The Journal of China Universities of Posts and Telecommunication, ELESVIER, 2014, pp. 67-73.

[14] Honglong Chen and Wei LouGAR, Group Aware Cooperative Routing Protocol For Resource-Constraint Opportunistic Networks, Computer Communications Elsevier, 2014, pp. 1-10.

[15] L.Wang, R.Wakikawa, R. Kuntz, R. Vuyyuru and L. Zhang, Data Naming in Vehicle-To-Vehicle Communications, in Proceedings of IEEE Conference on Computer CommunicationsWorkshops (INFOCOMWKSHPS), 2012, pp. 328-333.

[16] Md Abdul Alim, Xiang Li, Nam P. Nguyen and My T. Thai, Structural Vulnerability Assessment of Community-Based Routing in Opportunistic Networks, IEEE Transactions on Mobile Computing, DOI 10.1109/TMC.2016.2524571, pp. 1-15. 
[17] Konglin Zhua, Wenzhong, Xiaoming Fuc and Lin Zhanga, Data Routing Strategies In Opportunistic Mobile Social Networks: Taxonomy and Open Challenges, Computer Networks (2015), http://dx.doi.org/10.1016/j.comnet.2015.10.018, pp. 115 .

[18] PAN Da-ru, CAO Wei, LIU Xiong, SUN Jia-jia and SHI Xiao-jun, A Performance-Guarantee Routing Algorithm in Complex Distributed Opportunistic Networks, The Journal of China Universities of Posts and Telecommunications Elsevier, DOI: $10.1016 / \mathrm{S} 1005-8885(11) 60463-9$, pp. 87-93.

[19] Liu Yazhi, Niu Jianwei and Ma Jian, Content Encounter Probability based Message Forwarding in Opportunistic Networks, in Proceedings of IEEE International Conference on Information Science and Engineering, pp. 2589-2594.

[20] Shanshan Lu, Yanliang Liu, Yonghe Liu and Mohan Kumar, LOOP: A Location Based Routing Scheme for Opportunistic Networks, IEEE, 2012, pp. 118-126.

[21] Quoc Nguyen Viet, Pham Van Phuoc,Trinh Quoc Son and Lung Vu Duc, Social Routing: A Novel Routing Protocol for DelayTolerant Network based on Dynamic Connectivity, IEEE, 2015, pp. 1-6. 